\title{
Compressed Sensing Reconstruction of Radar Echo Signal Based on Fractional Fourier Transform and Improved Fast Iterative Shrinkage-Thresholding Algorithm
}

\author{
Rui Zhang ${ }^{D}$, Chen Meng $(D$, Cheng Wang, and Qiang Wang \\ Shijiazhuang Campus, Army Engineering University, Shijiazhuang 050003, China \\ Correspondence should be addressed to Chen Meng; 1096256108@qq.com
}

Received 12 April 2021; Revised 13 July 2021; Accepted 17 August 2021; Published 1 September 2021

Academic Editor: Telmo Reis Cunha

Copyright @ 2021 Rui Zhang et al. This is an open access article distributed under the Creative Commons Attribution License, which permits unrestricted use, distribution, and reproduction in any medium, provided the original work is properly cited.

\begin{abstract}
The compressed sensing theory, which has received great attention in the field of radar technology, can effectively reduce the data rate of high-resolution radar imaging systems and solve the problem of collecting, storing, and transmitting large amounts of data in radar systems. Through the study of radar signal processing theory, it can be found that the echo of radar LFM transmit signal has sparse characteristics in the distance upward; based on this, we can consider using the theory of compressed sensing in the processing of radar echo to optimize the processing. In this paper, a fast iterative shrinkage-thresholding reconstruction algorithm based on protection coefficients is proposed. Under the new scheme, firstly, the LFM echo signal's good sparse representation is obtained by using the time-frequency sparse characteristics of the LFM echo signal under the fractional Fourier transform; all reconstruction coefficients are analyzed in the iterative process. Then, the coefficients related to the feature will be protected from threshold shrinkage to reduce information loss. Finally, the effectiveness of the proposed method is verified through simulation experiments and application example analysis. The experimental results show that the reconstruction error of this method is lower and the reconstruction effect is better compared with the existing reconstruction algorithms.
\end{abstract}

\section{Introduction}

To improve the estimation accuracy of target parameters, it is necessary to increase the bandwidth of the radar, resulting in a significant increase in the amount of radar echo data storage, and transmission greatly increases. Linear Frequency Modulated (LFM) (also known as chirp) signals are widely used in sonar, radar, communication, and mine detection systems, etc. The analysis and processing of this signal have been a more important research direction in the field of modern signal processing [1-3]. According to Nyquist sampling theory, the data sampling must be at least twice the maximum frequency of the signal; in the face of high-frequency LFM signals, the sampling rate of existing high-speed ADC has been difficult to meet the requirements of Nyquist sampling, while the massive amount of data sampled also brings enormous pressure on data compression, transmission, and storage.
Compressed sensing (CS) $[4,5]$ is a new theory to solve the above problems, and its sampling frequency can be much lower than the Nyquist sampling rate. Under the compressed sensing scheme, data compression is performed simultaneously with sampling in the sensor. CS theory can be divided into three parts: sparse representation [6], measurement matrix, and reconstruction. First, only signals that are sparse enough in a certain transform domain can be considered compressible, which is the premise of CS application. Then, for the restricted isometric property (RIP) [7], the measurement matrix needs to be downsampled, which avoids the loss of information after the measurement. Some random matrices, such as Gaussian [8] and Bernoulli random matrices [9], have proven to be suitable for CS measurement. Finally, use an appropriate reconstruction algorithm to reconstruct the signal. Since the samples required for reconstruction are much lower than the Nyquist 


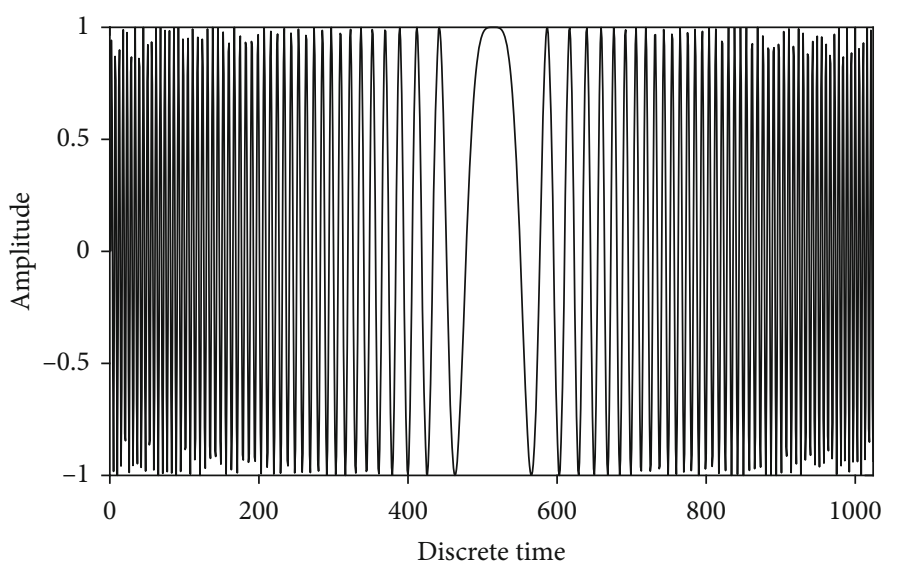

FIgure 1: The LFM echo signal.

sampling rate, compressed sensing reduces the sampling frequency and storage capacity in signal acquisition.

In the process of target detection, the radar should have a high target resolution, that is, a more accurate distinction between targets. To achieve this goal, we can expand the bandwidth of the radar transmit signal as required. Then, as the bandwidth of the transmit signal increases, it makes the data volume of the radar echo signal larger, which causes a certain burden to the storage and transmission of the echo data and will affect the real-time of the whole system work. Compressed sensing is widely used in the undersampling processing of LFM echo signals, and reconstruction is the most important part of compressed sensing; the performance of the reconstruction algorithm will directly affect the accuracy of the reconstruction results [10]. Currently, typical algorithms for solving this problem include the orthogonal matching pursuit algorithm (OMP) [11, 12], the regularized orthogonal matching pursuit algorithm (ROMP) [13], the Compressive Sampling Matching Pursuit algorithm (CoSaMP) [14], and the fast iterative shrinkagethresholding algorithm (FISTA) [15-17], among others.

The OMP algorithm and CoSaMP are simple and easy to implement with less computation, but they are prone to fall into local optima at low sampling rates and have large reconstruction errors. The ROMP algorithm can select multiple atoms in each iteration. Thus, the convergence speed of the algorithm is accelerated. However, they cannot guarantee that the selected atoms in each iteration are the correct ones. If the wrong atom was selected in the previous iteration, it will affect the selection of the atom in the next iteration.

FISTA is based on the idea of gradient descent and makes smarter choices in the iterative process, resulting in faster iterations. Using this method, the reconstruction calculation is simplified and the efficiency is improved. In view of the advantages of the FISTA algorithm in solving the reconstruction problem, the FISTA algorithm is used in this paper for the reconstruction process of compressed sampling of the time-frequency coefficients of LFM echo signals. However, when it is applied to the LFM echo signal, some new problems arise, one of which is poor reconstruction.
The acquired echo signal is usually mixed with noise. Then for FISTA, some useful information is lost during the iterative process, which leads to the poor reconstruction effect of FISTA and causes large reconstruction errors.

To solve this problem, an improved FISTA method is proposed in this paper for the compressed sensing reconstruction of LFM echo signals. Firstly, the sparse property of the LFM signal in the FRFT domain is used to establish an orthogonal dictionary to form a good sparse representation of the LFM signal. The reconstructed coefficients under the FRFT-based dictionary are analyzed during the iterative process of the FISTA algorithm. Then, the coefficients associated with the features are protected from threshold shrinkage to reduce information loss. Through the analysis of simulation experiments, the IFISTA algorithm proposed in this paper improves the reconstruction of LFM echo signals compared with using the traditional FISTA algorithm and can obtain higher reconstruction accuracy as well as higher reconstruction probability at lower SNR.

\section{Basics of LFM Signal Compressed Sensing Theory}

2.1. Basics of Compressed Sensing Theory. In compressed sensing theory, the signal acquisition process considers the sparsity and structural properties of the signal, and the whole process is represented as a "holographic" measurement. In this way, the signal is no longer limited by the bandwidth of the signal but is closely related to the information contained in the signal and can be compressed at the same time as the signal is acquired.

Suppose that $\left\{\boldsymbol{\Psi}_{i}\right\}_{i=1}^{N}$ is a set of basis vectors of $\mathbb{C}^{N}$ and $\mathbb{C}$ denotes a complex domain, then for any $\mathbf{x} \in \mathbb{C}^{N}, \mathbf{x}$ can be expressed as

$$
\mathbf{x}=\Psi s=\mathbf{s}_{1} \Psi_{1}+\mathbf{s}_{2} \Psi_{2}+\cdots \mathbf{s}_{N} \Psi_{N}
$$

where $\Psi=\left[\boldsymbol{\Psi}_{1}, \boldsymbol{\Psi}_{2}, \cdots, \boldsymbol{\Psi}_{N}\right]$ is a transformation matrix of $\left\{\Psi_{i}\right\}_{i=1}^{N}, \mathbf{s}$ is a sparse vector, and $\mathbf{s}_{i}=(i=1,2, \cdots, N)$ is the $i$ 


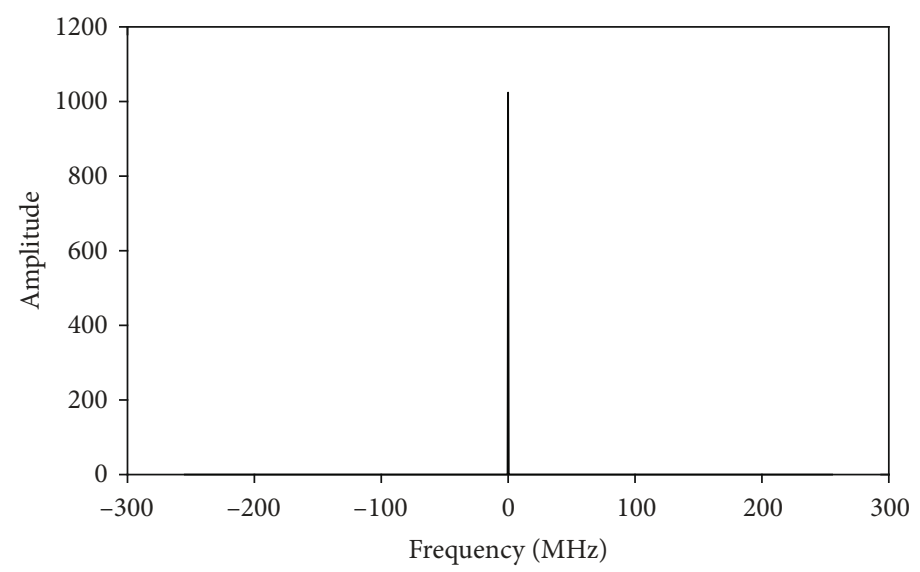

(a)

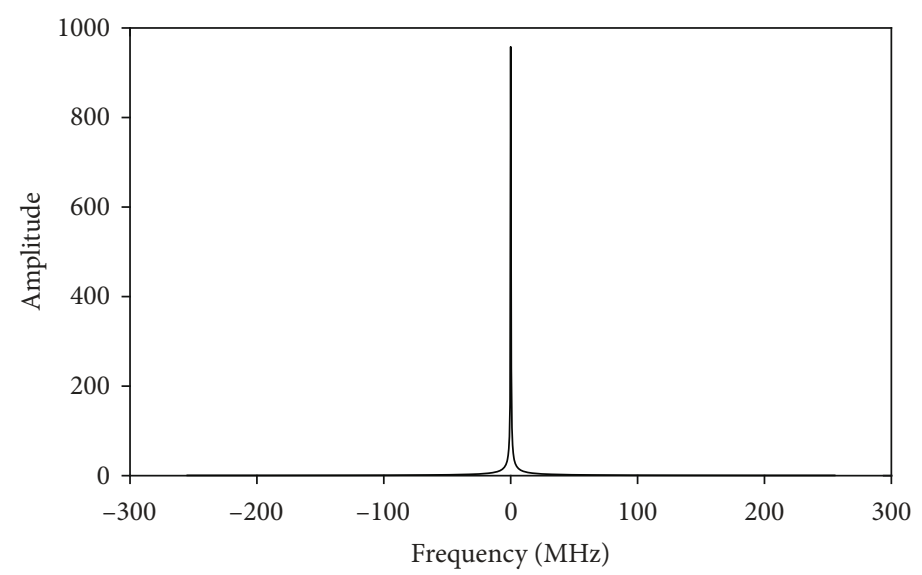

(b)

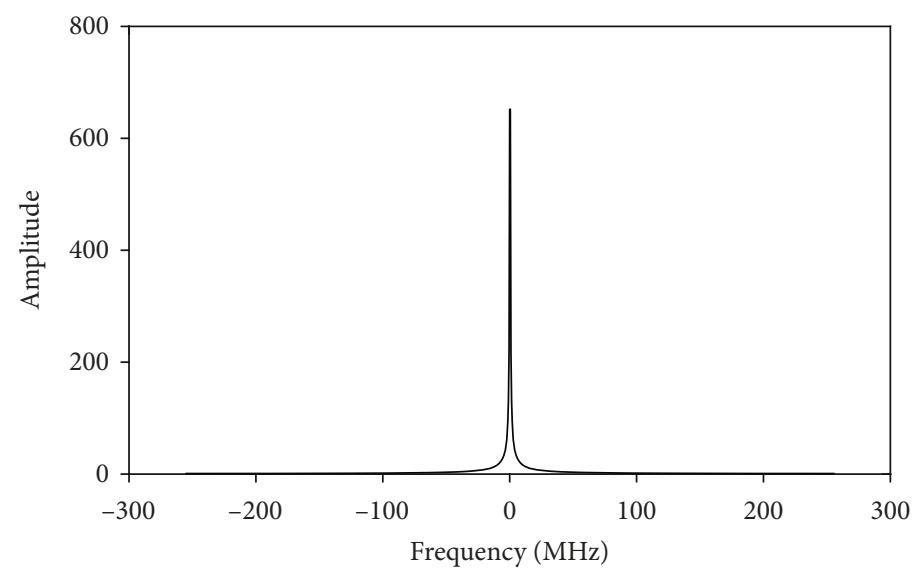

(c)

Figure 2: Fractional spectrum of LFM echo signals with different initial frequencies: (a) $f_{0}=0 \mathrm{MHz}$; (b) $f_{0}=0.1 \mathrm{MHz}$; (c) $f_{0}=$ $0.25 \mathrm{MHz}=0.5 \Delta f$.

th element of the vector $\mathbf{s}$. If the number of nonzero coefficients in $\mathbf{s}$ is $\mathbf{K}, K \in(1,2, \cdots, N)$, then $\mathbf{s}$ is said to be a $\mathbf{K}$ -sparse signal. Under compressive sensing theory, sparsity is a concrete expression of signal compressibility. Often, strictly zero coefficients are difficult to guarantee, so the $\mathbf{K}$ -term approximation is usually used to express the compressibility of the signal.
For $\mathbf{K}$-sparse signals, the process of compressing the measurement is expressed as

$$
\mathbf{y}=\Phi \mathbf{x}=\Phi \Psi^{\prime} \mathbf{s}=\Theta \mathbf{s}
$$

where $\Phi \in \mathbb{C}^{M \times N}$ is the observation matrix, $\Theta=\Phi \Psi$ is the 
TABLE 1: Leakage width values corresponding to different initial frequencies.

\begin{tabular}{lc}
\hline Initial frequency $(\mathrm{MHz})$ & Leakage width \\
\hline $0.1 \mathrm{MHz}$ & 20 \\
$0.15 \mathrm{MHz}$ & 30 \\
$0.25 \mathrm{MHz}$ & 50 \\
$0.30 \mathrm{MHz}$ & 40 \\
$0.50 \mathrm{MHz}$ & 0 \\
\hline
\end{tabular}

sensing factor, and $y \in \mathbb{C}^{M}$ is the observation vector. If the signal itself is compressible, then $\Psi$ is the unit array.

Compressed sensing enables the downscaling of the original signal through the measurement matrix, effectively reducing the number of signal points sampled. However, in order to accurately reconstruct the original signal, the sensing factor needs to satisfy the finite isometry property (RIP) to ensure that no useful information is lost during the downscaling observation process.

Since the observation vector dimension is smaller than the original signal dimension, the reconstruction process of the signal behaves pathologically. However, the compressibility of the signal in the transform domain offers the possibility of accurate reconstruction of the signal. The reconstructive model of the signal can be formulated as

$$
\mathbf{s}^{\prime}=\arg \min \|\mathbf{s}\|_{0} s t \quad \Theta \mathbf{s}=\mathbf{y},
$$

where $\|\cdot\|_{0}$ is the $l_{0}$ norm.

2.2. LFM Echo Signal Sparse Representation. In 1980, Namias first introduced the concept of fractional Fourier transforms based on arbitrary powers of eigenvalues from the perspective of eigenvalues and eigenfunctions of the Fourier transform [18], and in 1993, Ozaktas and Mendlovic applied the FRFT to optical implementations and optical information processing based on Mc Bride's work. In the same year, Almeida pointed out that the FRFT can be interpreted as a rotation of the time-frequency plane and that the FRFT is a class of linear time-frequency transforms in terms of the relationship between the time and frequency axes, which exhibit all the characteristics of a signal in both the time and frequency domains. In layman's terms, the Fourier transform of a signal can be thought of as a representation of the signal rotated counterclockwise from the time axis by $\pi / 2$ to the frequency axis, while its FRFT can be thought of as a representation of the signal rotated counterclockwise from the time axis by any angle to the $\mathbf{u}$ axis.

In [19], Ozaktas et al. give discrete algorithms with comparable complexity to FFT, making FRFT widely used in signal processing.

The FRFT of the signal $\mathbf{x}$ can be expressed as

$$
\begin{aligned}
X_{p}(u) & =\int_{-\infty}^{+\infty} K_{p}(u, t) x(t) d t, \\
K_{p}(u, t) & =A_{a} \exp \left[j \pi\left(u^{2} \cot \alpha-2 u t \csc \alpha+t^{2} \cot \alpha\right)\right] .
\end{aligned}
$$

$K_{p}(u, t)$ is the FRFT integral kernel function, where $A_{\alpha}=\sqrt{(1-j \cot \alpha)}, \alpha$ is the rotation angle and $\alpha=p \pi / 2$, and $p$ is the transform order; $K_{p}(u, t)$ can be regarded as a set of chirp signals with a modulation frequency of $\cot \alpha$, an initial frequency of $-u \csc \alpha$, and a complex envelope of $A_{\alpha}$. The set of chirp signals forms the orthogonal basis of the FRFT.

The modulation frequency of the chirp base varies with the rotation angle, and when the LFM signal coincides with the tuning frequency of one of the chirp bases, a $\delta$ function is generated, at which point the LFM signal shows good time-frequency aggregation characteristics in the FRFT domain. By finding the transform order of the LFM source signal that matches the energy aggregation property, the corresponding FRFT basis can be found and the corresponding dictionary of orthogonal sets can be constructed to achieve a good sparse representation of the signal.

In this paper, the Pei sampling algorithm is used to construct a dictionary of FRFT orthogonal bases. The basic idea is to directly sample the input and output variables and then maintain the invertibility of the transform by limiting the input and output sampling intervals. This algorithm is characterized by low computational complexity and high operational efficiency.

\section{The Improved FISTA for LFM Echo Signal Reconstruction}

3.1. FISTA Theory. For FISTA, solving the reconstruction problem can be described as

$$
s^{\prime}=\underset{s}{\operatorname{argmin}}\|\Theta \mathbf{s}-\mathbf{y}\|_{2}^{2} .
$$

However, if $\Theta$ is a pathological matrix, the condition number is larger in this case, and it is usually necessary to introduce regularization to solve the problem, where the $l_{1}$ regularization method is widely used to solve this problem.

$$
s^{\prime}=\underset{\mathbf{s}}{\operatorname{argmin}}\|\Theta \mathbf{s}-\mathbf{y}\|_{2}^{2}+\lambda\|\mathbf{s}\|_{1},
$$

where \|\|$_{1}$ is denoted as the $l_{1}$ parametric number, denoted as the sum of absolute values of $s$ elements. $l_{1}$ parametric number can be used to induce the optimal solution of sparsity. FISTA is an extension of the gradient descent method, where each iteration uses the information from the current point for iterative estimation and updates the iteration points. Its iterative form can be expressed as follows:

$$
s^{(n+1)}=\mathscr{T}_{\alpha}\left(s^{(n)}-2 t^{(n)} \Theta^{T}\left(\Theta s^{(n)}-y\right)\right),
$$

where $t^{(n)}>0$ is the step size, which generally requires that $t^{(n)} \in\left(0,1 /\|\Theta\|_{2}^{2}\right)$ and $\mathscr{T}_{\alpha}$ is the shrinkage operator.

$$
\mathscr{T}_{\alpha}\left(s_{i}\right)=\left(\left|s_{i}\right|-\alpha\right)_{+} \operatorname{sgn}\left(s_{i}\right) .
$$

|| is the absolute value, ()$_{+}$is expressed as the maximum 
Input: the compressed sampling signal $y$, the measurement matrix $\Phi$, the DFRFT basis dictionary $\Psi$, the regularization parameter number $\lambda$

Output: reconstructed signal $s^{\prime}$

1.Gradient step $G^{n}=s^{(n)}-2 t^{(n)} \Theta^{T}\left(\Theta s^{(n)}-y\right)$;

2. Shrinkage threshold $s^{(n+1)}=\mathscr{T}_{\alpha}\left(G^{n}\right)$

3. Select the eigencoefficient, generate the index vector eigencoefficient $\Lambda$

4. Protect the vector $\Lambda$ from shrinkage

5. Iteration step, meet the iteration stop condition, then return the reconstructed signal $s^{\prime}$

Algorithm 1: The improved FISTA for LFM signal.

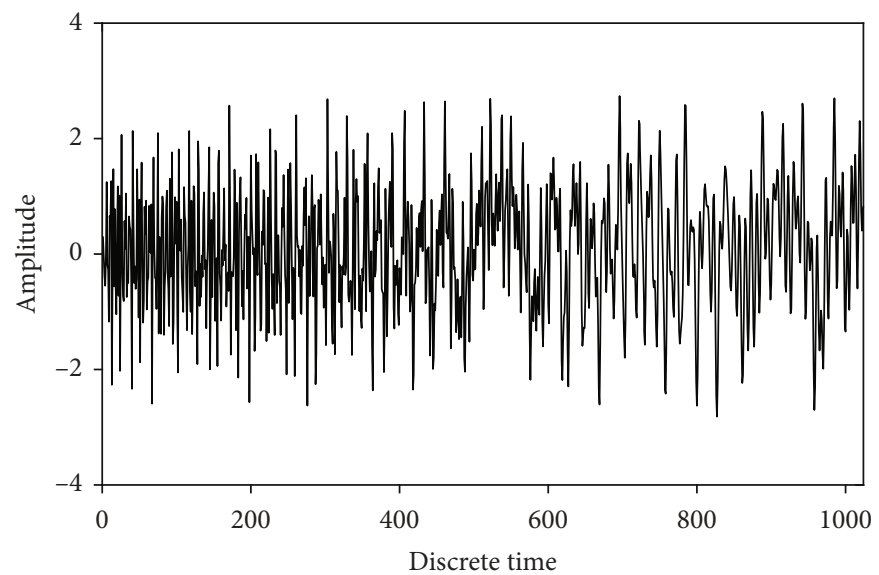

(a)

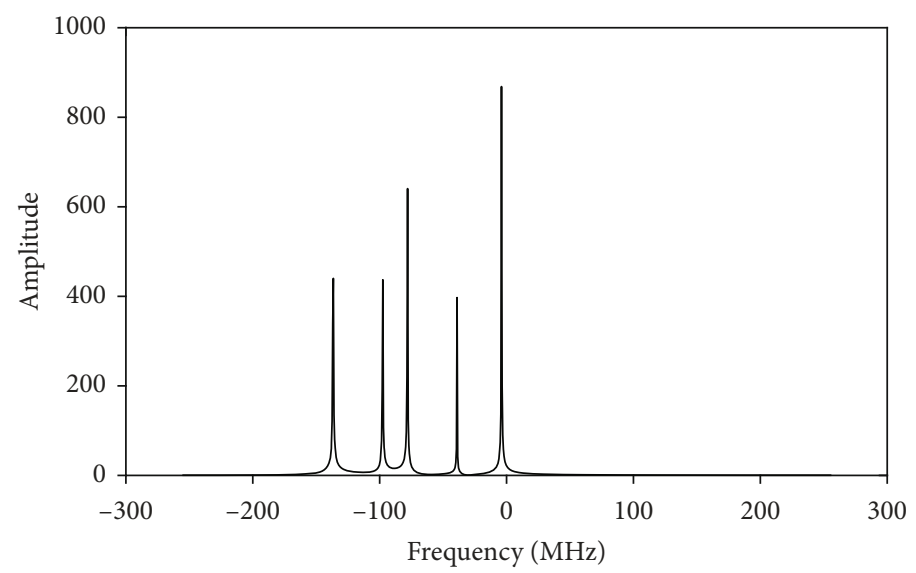

(b)

Figure 3: The simulated signals: (a) time-domain waveform of LFM echo signals; (b) fractional spectrum of LFM echo signals.

value among $\left|s_{i}\right|-\alpha$ and 0 , and sgn is the sign function, indicating that if $s_{i}$ is greater than 0 , sgn turns 1 ; equal to 0 , it returns 0 ; less than 0 , it returns -1 .

\subsection{The Analysis of FISTA When Applied to LFM Echo} Signals. FISTA is computationally simple, converges quickly, and takes less time in the reconstruction process. However, when applied to LFM signals, the reconstruction effect needs to be further improved. For LFM signals, its reconstruction coefficients contain some small-value coefficients. These useful small-value coefficients may be discarded during the iterative process, resulting in the loss of information in the reconstruction.

The shrinkage threshold can be divided into two substeps: one is the gradient step, which can be described as

$$
G^{n}=s^{(n)}-2 t^{(n)} \Theta^{T}\left(\Theta s^{(n)}-y\right) .
$$




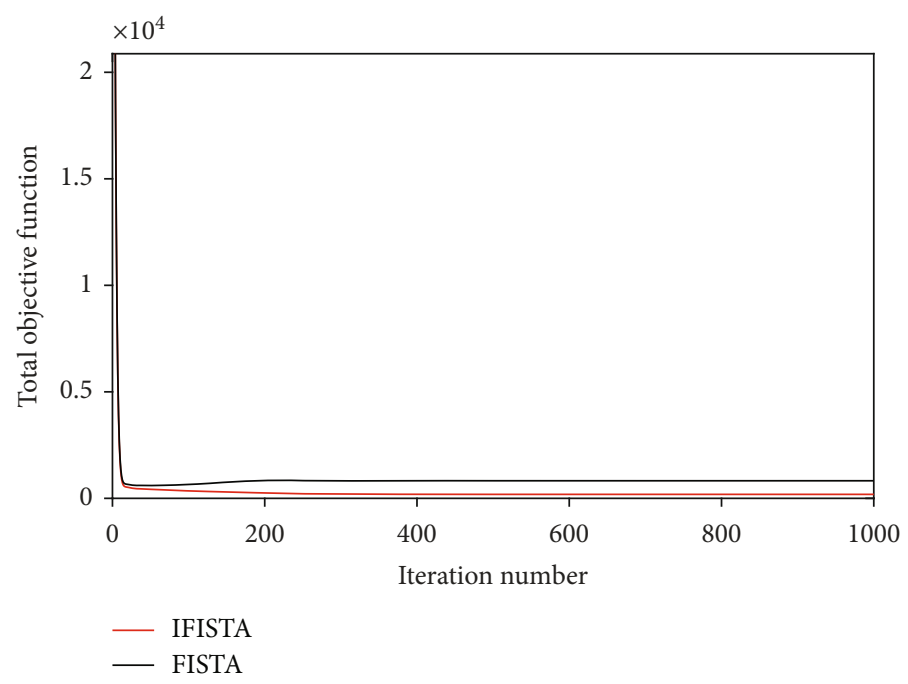

(a)

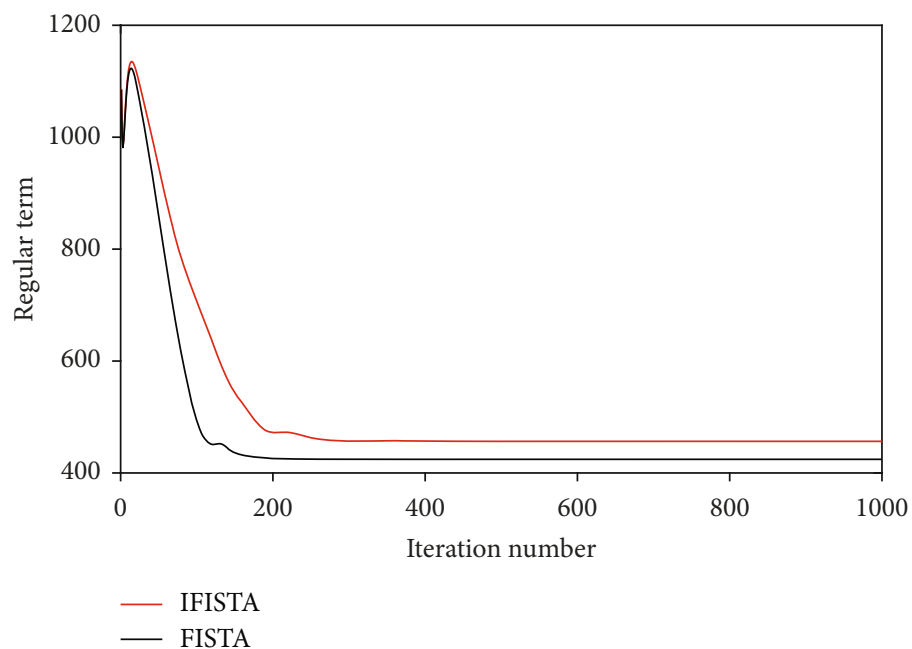

(b)

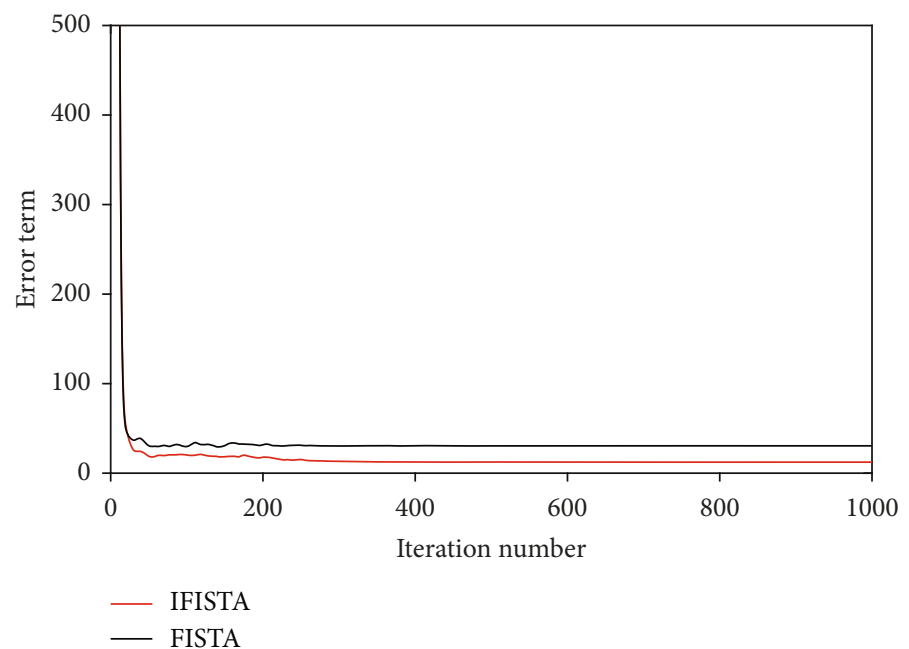

(c)

FIGURE 4: The relation between the objective function and the number of iterations: (a) the total objective function; (b) the regular term; (c) the error term. 


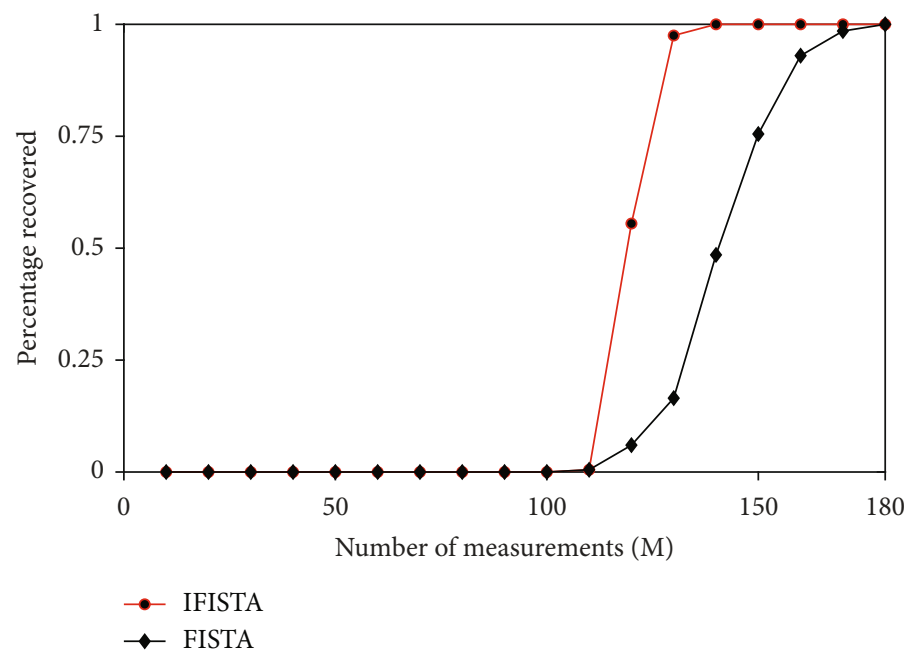

(a)

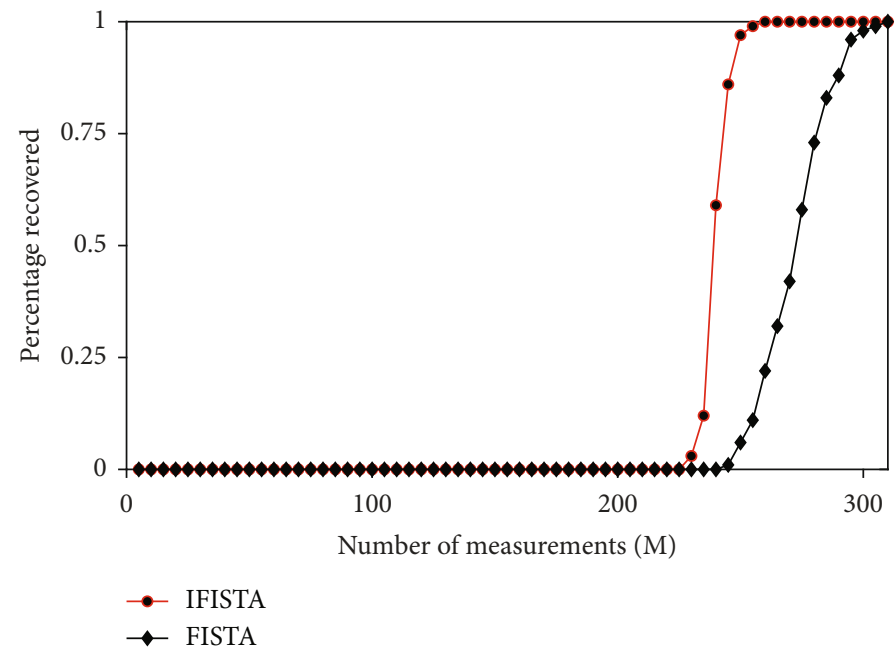

(b)

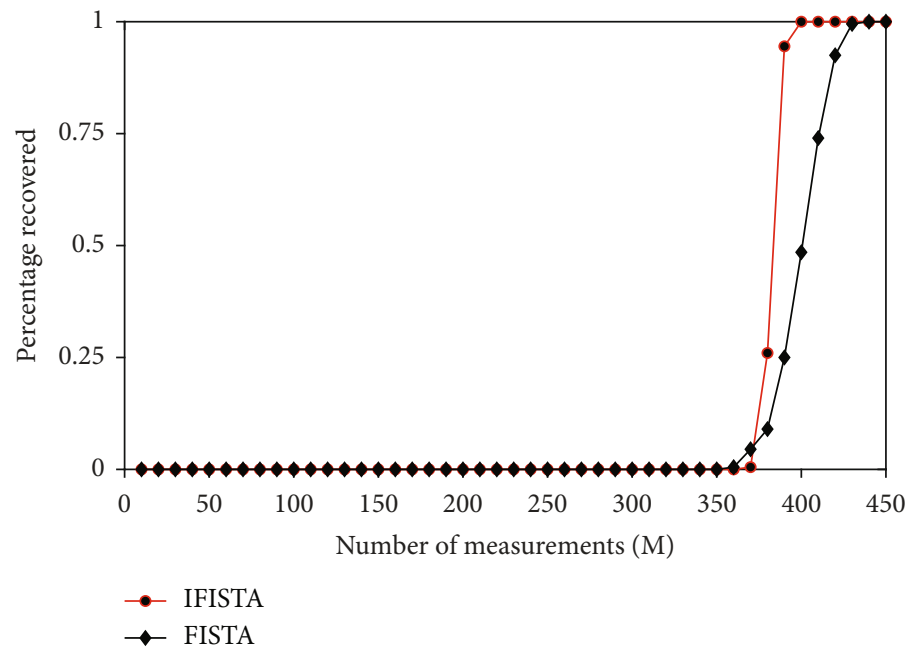

(c)

FIGURE 5: The relationship between the success rate of reconstruction and the number of sampling points under different sparsity: (a) $K=k_{0}$; (b) $K=3 k_{0}$; (c) $K=5 k_{0}$. 


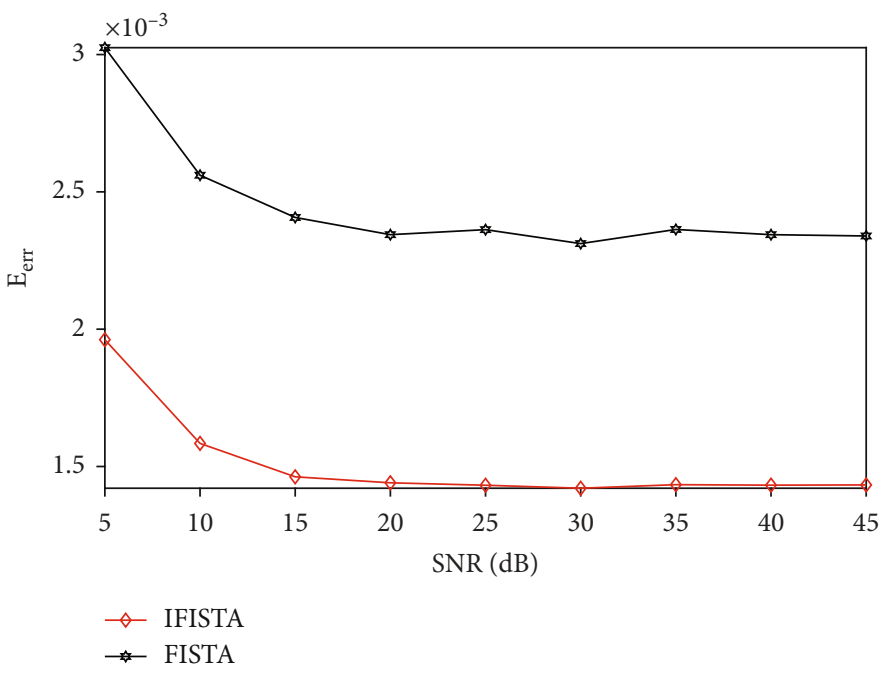

(a)

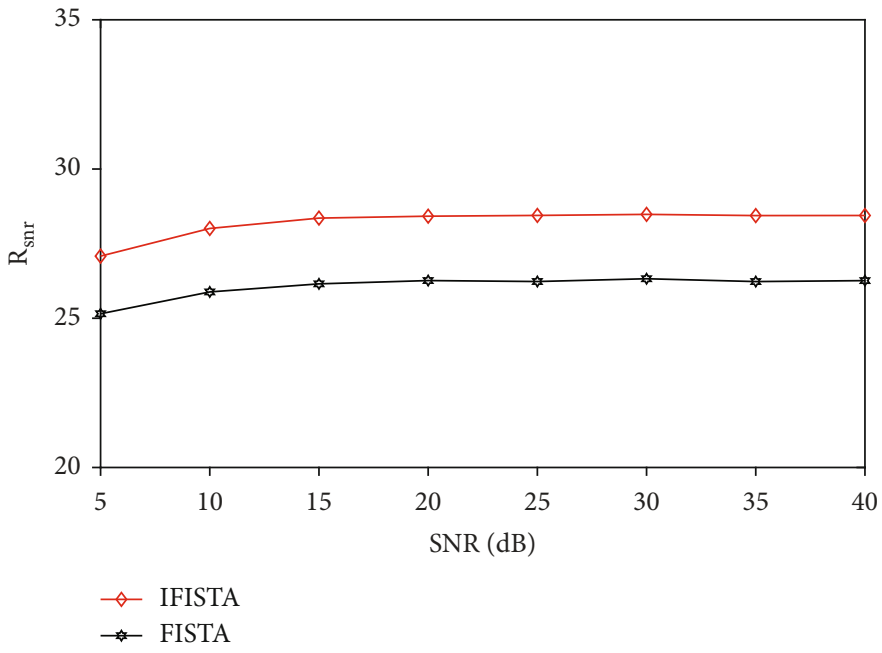

(b)

FIGURE 6: The change tendency of reconstruction performance evaluation criterion with SNR: (a) reconstruction error; (b) reconstructed signal-to-noise ratio.

Another substep is the shrinkage step using the shrinkage operator

$$
s^{(n+1)}=\mathscr{T}_{\alpha}\left(G^{n}\right) .
$$

The gradient step is used to minimize the reconstruction error, and the shrinkage operator is aimed at approximating the satisfaction of the canonical term. For the shrinkage operator $\mathscr{T}_{\alpha}$, it is known from (16) that is an extrapolation of all elements in $x$, after which the elements are retained if they are still positive and will be 0 if they are negative.

The usually obtained LFM echo signal will be contaminated with noise because of the complexity of the target environment. Then, going through the gradient step may lead to bias and thus mislead the iterative process, which in turn causes a decrease in the iteration speed. On the other hand, when using the shrinkage operator, each sparse coefficient of the LFM signal over the FRFT domain is subtracted from $\alpha$. Then, for some small useful coefficients, this method brings a loss of information, which can lead to an increase in reconstruction error. And the information loss is brought by the shrinkage operator, then the increase in the number of iteration steps caused by the noise will further increase the error.

3.3. The Improved FISTA for LFM Echo Signal. In order to improve the reconstruction of LFM echo signal, IFISTA is proposed in this paper. Under the proposed scheme, the coefficients considered as features do not need to participate in the next iteration of the shrinkage. These coefficients will then help to retain useful information in the reconstruction. The key of the scheme is how to choose the feature coefficients.

The LFM echo signal can be expressed as

$$
x(t)=A \exp \left[j\left(2 \pi f_{0} t+\pi k t^{2}\right)\right],
$$




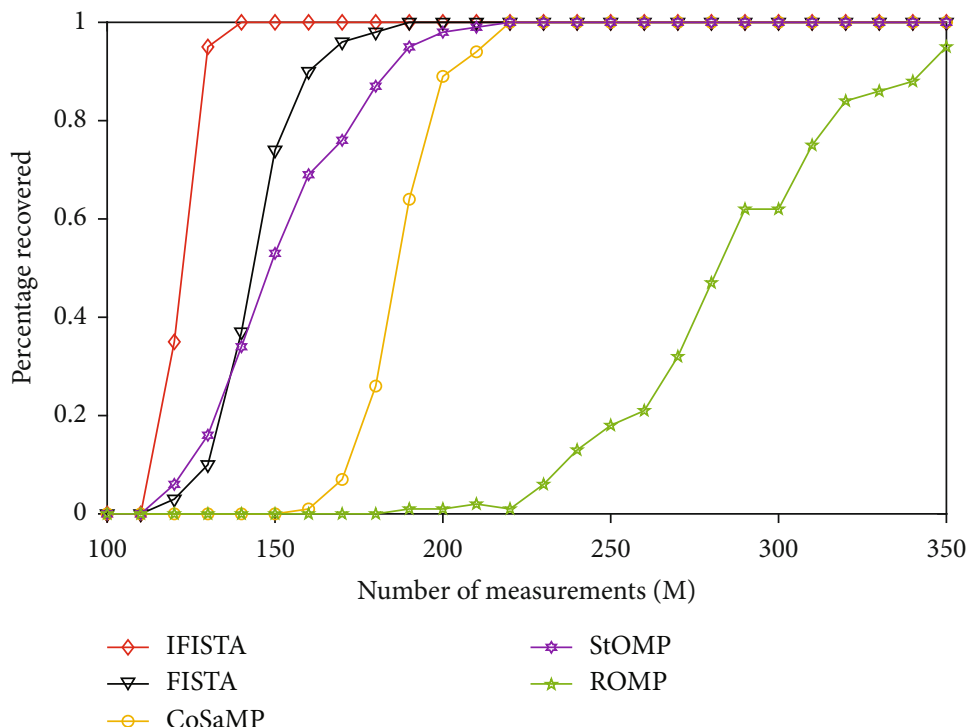

(a)

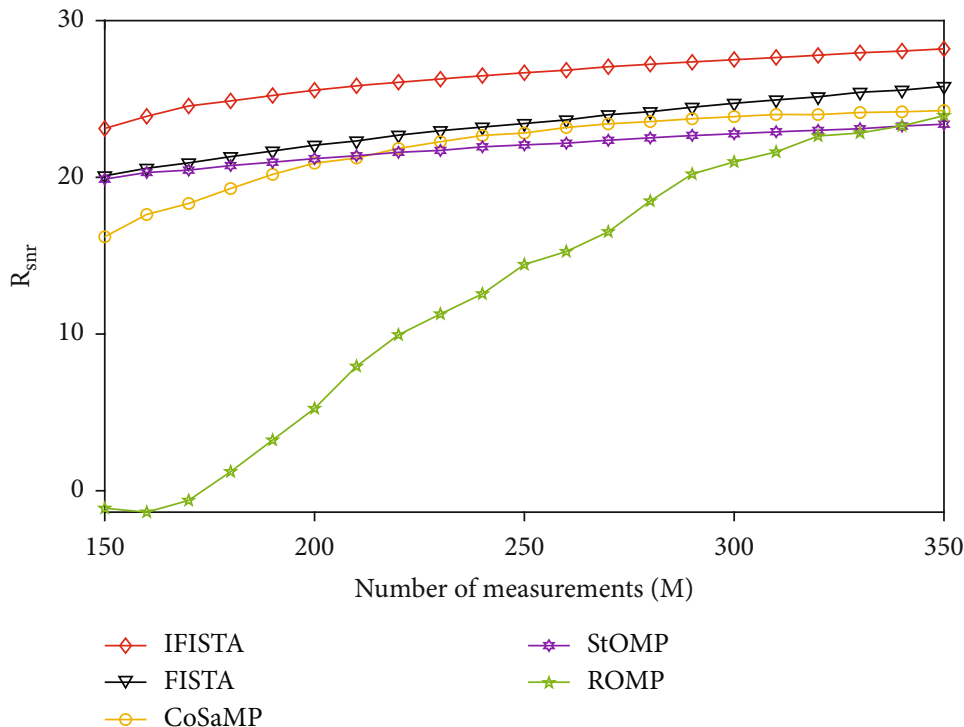

(b)

FigURE 7: The relationship between the reconstruction performance and the number of measurements $(M)$ : (a) percentage recovered; (b) reconstructed signal-to-noise ratio.

where $A$ is the amplitude of the signal, $f_{0}$ is the initial frequency of the signal, and $k$ is the modulation frequency; the simulated time-domain waveform of the LFM signal is shown in Figure 1.

The sampling frequency is $f_{s}=512 \mathrm{MHz}$, the number of sampling points is 1024, and the frequency resolution is $\Delta f=f s / N=0.5 \mathrm{MHz}$. Figure 2 shows that the LFM signal has good sparsity in the fractional-order spectrum. When the initial frequency of the simulated LFM signal does not satisfy an integer multiple of the frequency resolution, the fractional-order spectrum will produce leakage, as shown in Figures 2(b) and 2(c).

The fractional-order spectrum contains both largevalued coefficients and certain small-valued coefficients, and these small-valued coefficients may be discarded during the iterative process of useful small-valued coefficients, which leads to the loss of information in the reconstruction. To solve this problem, indexing the eigencoefficient vector in the fractional-order spectrum is proposed to obtain all the eigencoefficients.

As shown in Table 1, when the initial frequency is an integer multiple of the frequency resolution, the fractionalorder spectrum has no leakage, the width of leakage is 0 , and the fractional-order spectrum contains only large-value coefficients; when the initial frequency is $1 / 2$ of the frequency resolution, the width of leakage generated in the fractional-order spectrum is the largest, and the smallvalue coefficients are the most numerous. 
A critical condition of $1 / 2$ times the frequency resolution is used to determine the range of the indexed eigencoefficient vector in the fractional-order spectrum. The index range is determined by keeping the coefficients at 0.01 times of the maximum coefficients, which can better avoid the loss of reconstruction information.

$$
\begin{aligned}
D\left(e^{j \omega_{0}}\right) & =0.01 N, \\
\frac{N_{0}}{N} \times 2 \pi & =\omega_{0},\left(N_{0}=0.01 N\right) .
\end{aligned}
$$

The fractional-order spectral positions of the atoms $\omega_{0}$ are determined to obtain the extended range $\Delta n$, and then, the index eigencoefficient vector $\Lambda$ is obtained.

To improve the FISTA for LFM signal compressed sensing, a simple analysis of the unstable reconstructed signal during the iteration process was carried out. As the iteration proceeds, the signal is gradually reconstructed to approximate the original signal, and therefore, it is feasible to extract information during the iterative process. Under the proposed IFISTA scheme, the reconstructed signal is analyzed during the fractional-order spectrum iteration. As mentioned above, the acquired LFM signal usually has two characteristic coefficients, a large-valued coefficient and a small-valued coefficient determined by the index vector. The coefficients belonging to both of these cases are then considered to be eigencoefficients, and these eigencoefficients do not participate in the shrinkage step of the shrinkage operator $\mathscr{T}_{\alpha}$, thus enhancing the characteristics of the reconstructed signal and improving the reconstructed effect.

We determined the range of coefficients to be protected by analyzing the sparse coefficients of the LFM signal in the fractional-order Fourier variable domain and obtained the index vector eigencoefficient $\Lambda$. First, obtain the fractional-order spectrum of the original signal under the fractional order Fourier transform dictionary, then find the location of the spectral peak that generated the shock $f_{i}$; the eigencoefficient is the small-value coefficient around the spectral peak, ranging from $\Delta n$ as determined above; then, the index vector eigencoefficient $\Lambda=\left\{\left(f_{1}-\Delta n, f_{1}+\Delta n\right)\right.$, $\left.\left(f_{2}-\Delta n, f_{2}+\Delta n\right), \cdots,\left(f_{i}-\Delta n, f_{i}+\Delta n\right)\right\}$ can be obtained. Then, we use $\Lambda$ to improve the FISTA as shown in Algorithm 1.

\section{Experimental Results and Discussion}

To verify the effectiveness of the method, we conducted simulation experiments. The simulated LFM echo signal model can be expressed as follows:

$S t=\sum_{i=1}^{K} A \exp \left\{j 2 \pi\left[f_{0}\left(t-\tau_{i}\right)+\frac{1}{2} k\left(t-\tau_{i}\right)^{2}\right]\right\}, \quad t \in[0, T]$.

$f_{0}$ is the carrier frequency, $T$ is the pulse time bandwidth, $k$ is the modulation frequency, $K$ is the number of scattering points, in the FRFT base dictionary, that is, the sparsity of
TABle 2: Calculation time for different algorithms.

\begin{tabular}{lccc}
\hline Method & \multicolumn{3}{c}{ Sampling rate } \\
& 0.3 & 0.5 & 0.7 \\
\hline IFISTA & 1.160 & 1.417 & 1.642 \\
FISTA & 0.987 & 1.160 & 1.422 \\
CoSaMP & 5.531 & 7.074 & 8.138 \\
StOMP & 3.420 & 5.403 & 7.280 \\
ROMP & 4,273 & 5.740 & 6.140 \\
\hline
\end{tabular}

TABle 3: Measured data parameters.

\begin{tabular}{lc}
\hline Parameter name & Numerical value \\
\hline Radar sampling frequency & $32.317 \mathrm{MHz}$ \\
Radar centre frequency & $5.3 \mathrm{GHz}$ \\
Azimuthal sampling frequency & $1256.98 \mathrm{~Hz}$ \\
Radar pulse tuning frequency & $721.35 \mathrm{MHz} / \mathrm{s}$ \\
Azimuthal tuning frequency & $1733 \mathrm{~Hz} / \mathrm{s}$ \\
\hline
\end{tabular}

the echo signal, and $A_{i}$ and $\tau_{i}$ denote the scattering intensity and time delay of the $i$ scattering point, respectively.

The simulated signal parameters are set as $f_{0}=0, B=$ $200 \mathrm{MHz}, T=2 \mu \mathrm{s}$, the sampling rate $f_{s}=512 \mathrm{MHz}$, the time delay $\tau=1 / f_{s}$, the length of the signal is 1024, the LFM signal with 5 components is set with the distance starting position $20 \tau, 200 \tau, 400 \tau, 500 \tau$, and $700 \tau$, and the normalized amplitude of the signal is $0.9,0.4,0.7,0.5$, and 0.6 , respectively. A DFRFT orthogonal basis dictionary $\Psi$ of the signal was constructed using a Pei sampling-type algorithm, using a Gaussian random matrix as the measurement matrix. The time-domain waveform of the analog signal is shown in Figure 3(a), and its fractional-order spectrum is shown in Figure 3(b).

Case 1. Comparison of objective function with the iteration.

Using the measurement matrix, the signal is sampled at a lower dimension than the source signal. In this case, we set the compression rate to 0.5 , at which point the length of the measurement vector is 512 . $\lambda$ is empirically set to 0.02 , when the iteration works best. The proposed IFISTA is compared with the FISTA proposed in [15].

It can be seen from Figure 4 that the error term $\left(\|\Theta \mathbf{s}-\mathbf{y}\|_{2}^{2}\right)$, the regular term $\left(\lambda\|\mathbf{s}\|_{1}\right)$, and the total objective function $\left(\|\Theta \mathbf{s}-\mathbf{y}\|_{2}^{2}+\lambda\|\mathbf{s}\|_{1}\right)$ are decreasing as the number of iterations increases. As shown in Figure 4(b), after the completion of the iteration, the final result using IFISTA's regular term is higher than using FISTA, this is due to the fact that using the proposed IFISTA, the eigencoefficients are not affected by shrinkage, thus increasing the rule term. Then, from Figure 4(c), the error term of IFISTA is lower than FISTA after the completion of the iteration, which indicates that the error of IFISTA is less than FISTA, which means that the proposed IFISTA reduces the reconstruction error. The IFISTA proposed in this paper can reconstruct the LFM echo signals more perfectly and get more useful information. 


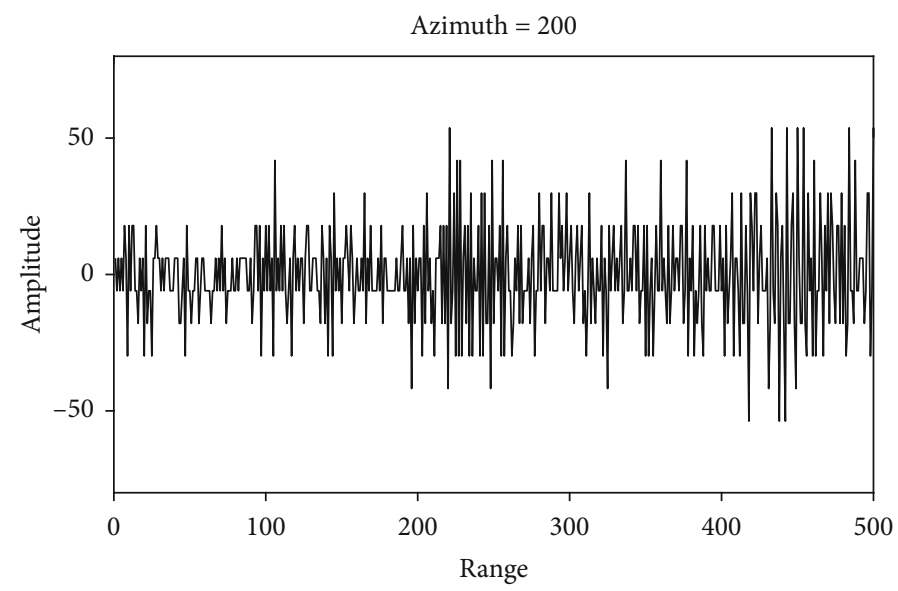

(a)

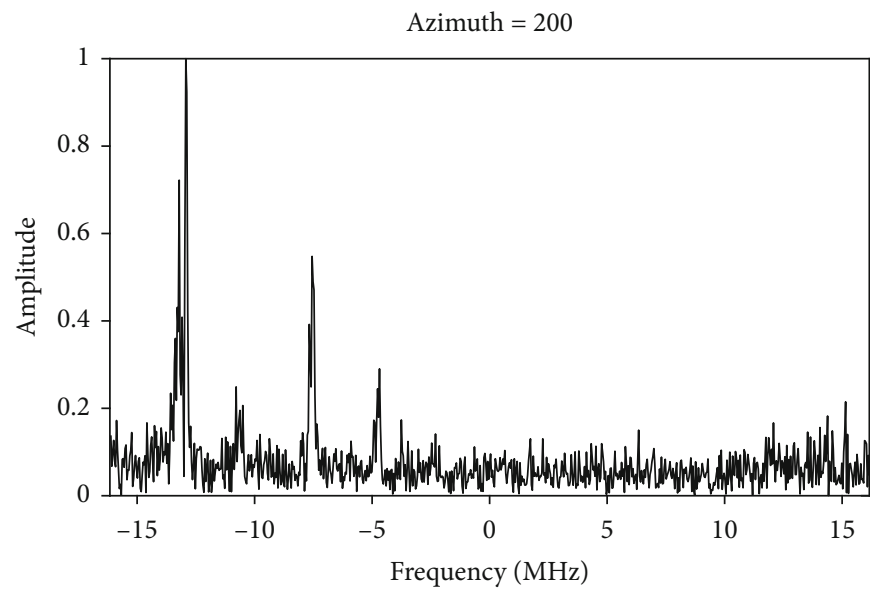

(b)

Figure 8: Measured echo signals: (a) waveform for real echo signals at the azimuth position 200; (b) fractional spectrum of real echo signals at the azimuth position 200 .

Case 2. Comparison of the percentage of exact reconstruction with different sparsity.

The reconstruction error a $E_{\text {err }}=\left\|s^{\prime}-s\right\|_{2}^{2} /\|s\|_{2}^{2}$ is defined to characterize the recovery performance of the algorithm, $s$ is the sparse coefficient of the signal under the FRFT base dictionary, $s^{\prime}$ is the sparse coefficient of the reconstruction, if the reconstruction error is less than the specified threshold $T_{\text {tol }}\left(T_{\text {tol }}=0.01\right)$, the reconstruction is considered successful, 1000 Monte-Carlo experiments are done, and the number of successful reconstructions is recorded as $n_{\text {suc }}$; then, the reconstruction success rate is $n_{\text {suc }} / 1000$.

According to the theory of compressed sensing, the sparsity of the signal is an important factor affecting the signal reconstruction effect. To further measure the performance of the proposed algorithm, the proposed IFISTA and FISTA reconstruction effects are compared for signals with different sparsity. The simulated LFM echo signals are set as 1-component echo signal, 3-component echo signals, and 5-component echo signals, respectively, and the sparsity of the single-component LFM signal is defined as $k_{0}$, and the sparsity of the three selected signals are $k_{0}, 3 k_{0}$, and $5 k_{0}$, respectively.
The comparison of IFISTA and FISTA reconstruction performance was carried out under different sampling rate conditions. From Figure 5, it can be seen that the probability of successful signal reconstruction increases continuously with the increase of sampling points. Compared with FISTA, the proposed IFISTA can reconstruct successfully with fewer sampling points. At the same time, as the sparsity of the signal increases, the sparsity of the signal becomes worse, more sampling points are required for successful reconstruction, and higher sampling frequency is required. However, compared to FISTA, IFISTA can still reconstruct the signal successfully with fewer sampling points. This means that IFISTA can have a lower sampling rate than FISTA with a stable reconstruction. This is of great interest for practical applications, since for the proposed IFISTA, ideal reconstruction requires fewer measurements.

Case 3. Comparison of the reconstruction performance in the noise case.

To analyze the effect of noise on the reconstruction method in this paper, noise with a different signal-to-noise 


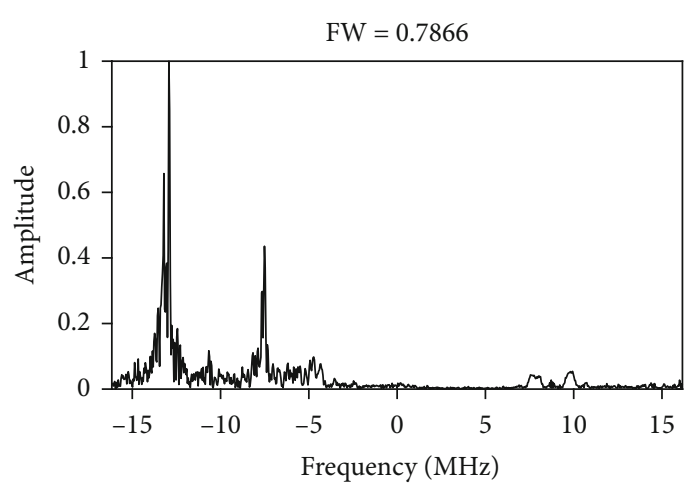

(a)

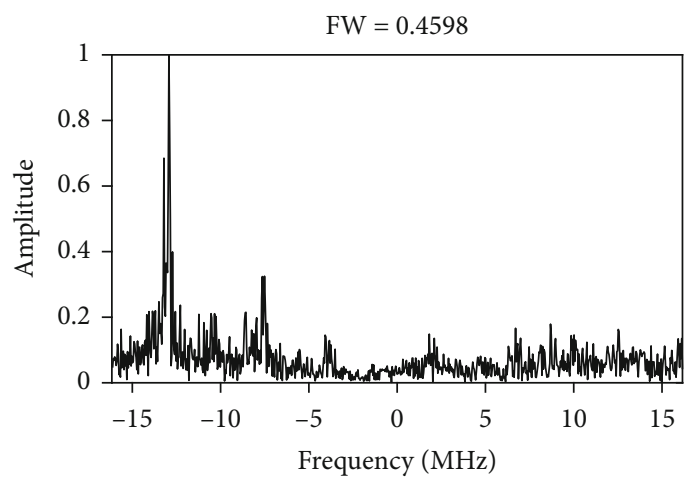

(c)

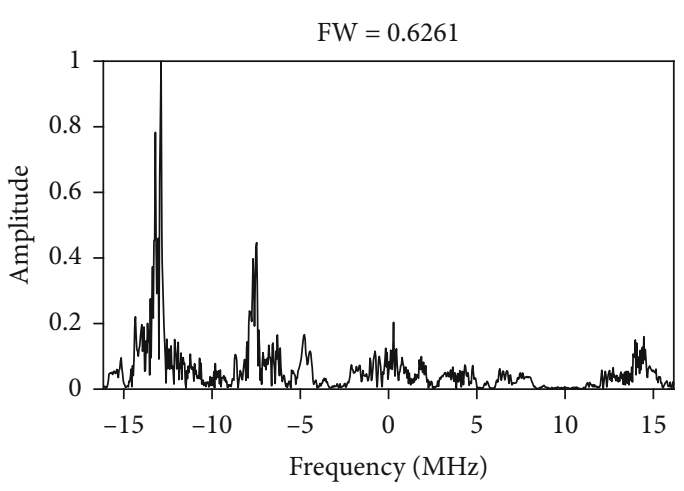

(b)

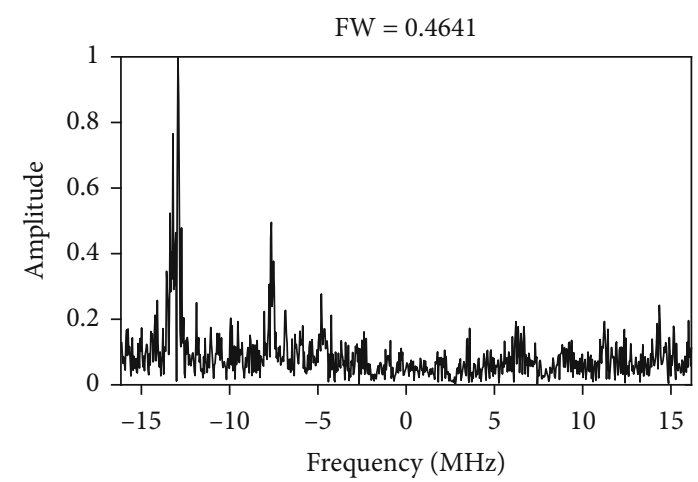

(d)

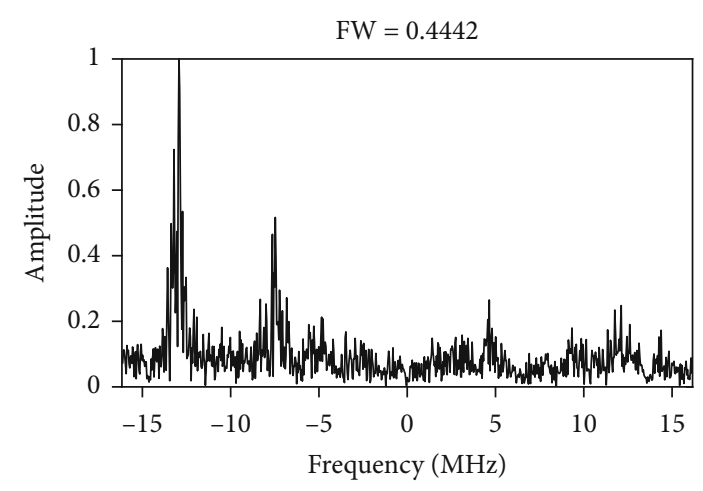

(e)

Figure 9: Fractional spectrum of reconstructed signals for different algorithms: (a) IFISTA; (b) FISTA; (c) CoSaMP; (d) StOMP; (e) ROMP.

ratio (SNR) is added to the compressed sampled signal. Define the reconstructed signal-to-noise ratio of the signal $R_{\mathrm{snr}}=10 \cdot \log _{10}\left(\left\|s^{\prime}\right\|_{2}^{2} /\left\|s^{\prime}\right\|-\|s\|_{2}^{2}\right)$ as reconstruction performance evaluation criterion. We performed CS experiments by fixing $M=370, K=k_{0}$, and varying the SNR from 5 to 45 , with the added noise being Gaussian white noise.

From Figure 6(a), we can see that the reconstruction error decreases as the signal-to-noise ratio increases. When the SNR changes from 5 to 25 , the reconstruction error decreases more obviously, and when the SNR changes from 25 to 45, because at this time the signal-to-noise ratio is already relatively large and the noise content is less, the reconstruction error basically tends to stabilize. Meanwhile, the reconstruction error of the method proposed in this paper is always lower than that of the FISTA algorithm proposed in [15]. From Figure 6(b), the signal-to-noise ratio of the reconstructed signal is improved when the added SNR is varied from 5 to 15 . The reconstructed signal-to-noise ratio stabilizes as the SNR varies from 15 to 40. The reconstructed signal-to-noise ratio of the proposed algorithm in this paper is always higher than that of FISTA.

With the increase of SNR, the reconstruction performance of the algorithms is improved. The algorithm in this paper protects the useful coefficients in the reconstructing process and prevents some feature coefficients from shrinking, which makes the reconstructing error lower and the higher reconstructed signal-to-noise ratio, and the IFISTA proposed in this paper gets better reconstruction performance than the FISTA algorithm.

Case 4. Comparison of the reconstruction performance with other algorithms. 


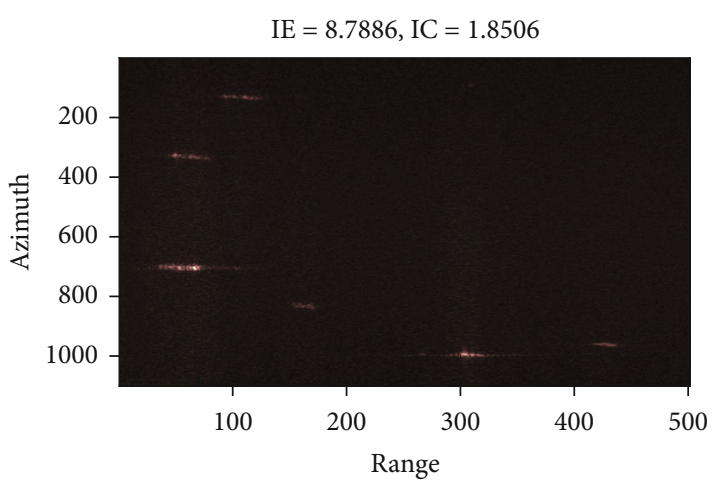

(a)

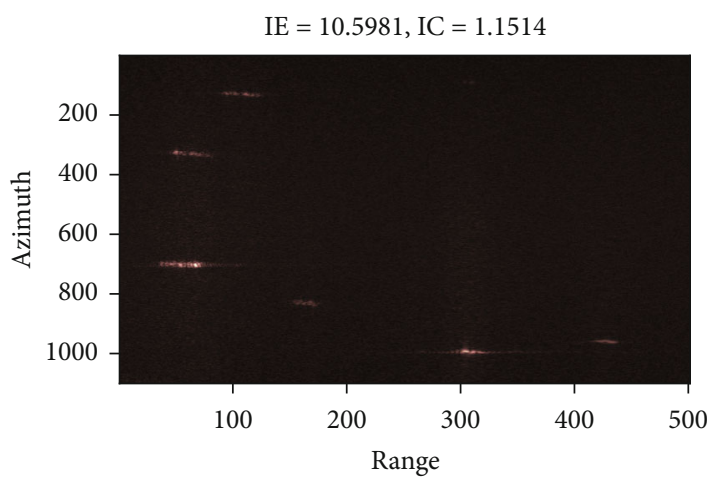

(c)

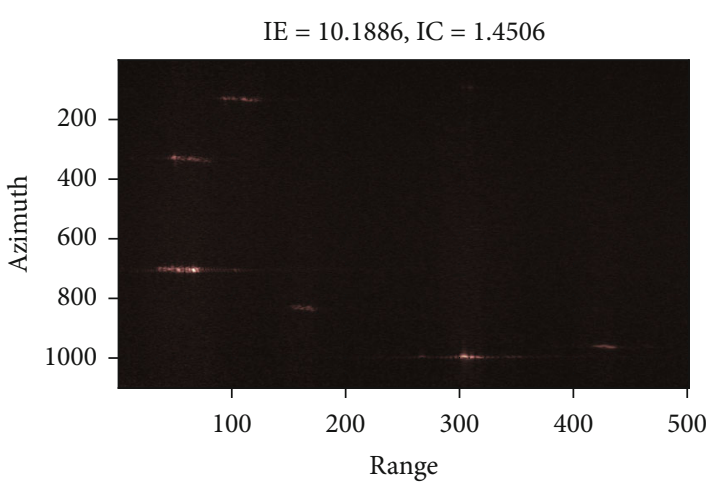

(b)

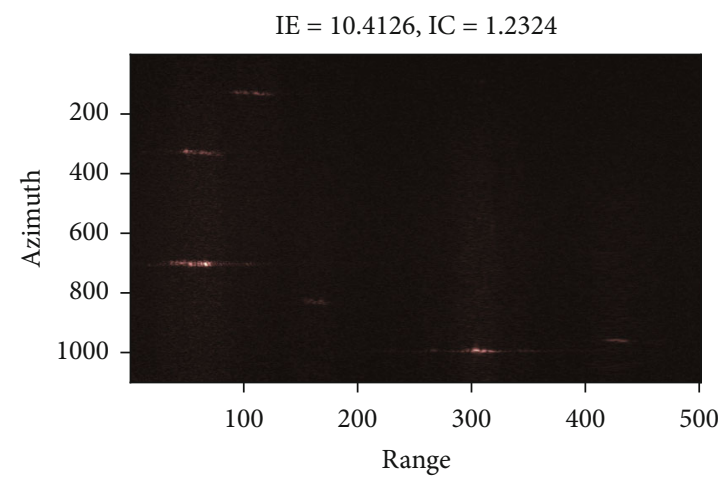

(d)

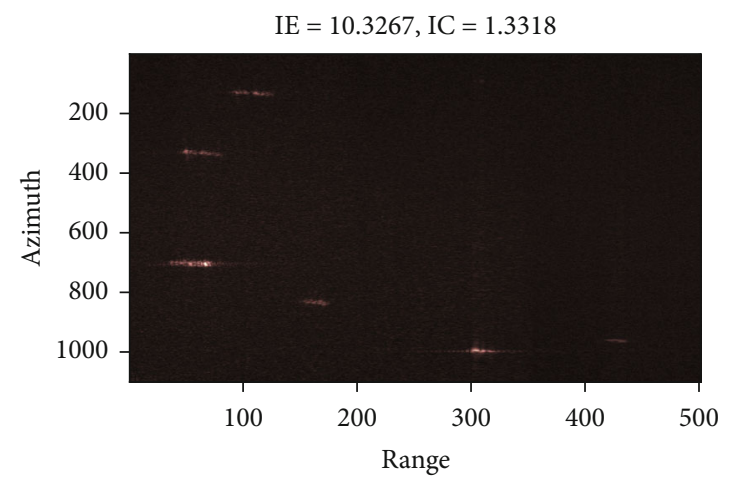

(e)

FIgure 10: Comparison of imaging results: (a) IFISTA; (b) FISTA; (c) CoSaMP; (d) StOMP; (e) ROMP.

The performance of the IFISTA was compared to other typical reconstruction algorithms, such as the CoSaMP, StOMP, ROMP, and FISTA. In this case, the simulated signal is set as a single component echo signal with sparsity $K=k_{0}$ and varies $M$ from 100 to 350 .

Figure 7 (a) illustrates the change tendency of the reconstruction success rate with parameter $M$ for $K=k_{0}$. It can be seen from the figure that the reconstruction success rate increases with $M$. The reconstruction performance of IFISTA outperforms other reconstruction algorithms. In general, for LFM echo signals, the number of observations required to stably reconstruct the signal using the method proposed in this paper is less than the FISTA, CoSaMP, StOMP, and ROMP algorithms. Figure 7(b) shows that, with the increase of $M$, the reconstructed signal-to-noise ratio is improved, and the $R_{\mathrm{snr}}$ of the proposed method is always higher than other algorithms. Using the IFISTA has some improvement for reconstruction accuracy.

Table 2 records a comparison of the time at which the optimal reconstruction is obtained by different algorithms for signals at different sampling rates. As the sampling rate increases, the reconstruction time increases and the reconstruction results improve significantly, but the IFISTA and FISTA algorithms take significantly less time to run than the other three algorithms, and the reconstruction results also improve. As the sampling rate increases, the number of sampling points increases; the computation time of the other three algorithms increases a lot, while the computation time of IFISTA and FISTA remains basically the same. IFISTA has a slight increase in computation time compared 
to FISTA, but the reconstruction accuracy is greatly improved, and the computation time is still much smaller than the other three algorithms.

Case 5. Comparison of the reconstruction performance with real LFM echo signal.

In order to further verify the effectiveness of the algorithm method proposed in this paper, the SAR radar echo signal is selected as an application example for the numerical analysis experiments of compressed sensing reconstruction of the SAR radar echo signal, which is a typical LFM echo signal. In this paper, a scenario from the RADARSAT-1 measured data [20] was used to verify the validity of the method in this paper. The scenario is a harbour in Vancouver, Canada, with six cargo ships docked. The parameters related to the measured data are shown in Table 3 .

The total sampling time is set to $15.47 u s$ and $0.7956 \mathrm{~s}$ for the distance and azimuth directions, respectively. The measured data dimension for this scene is $500 \times 1000$. The measured signals are shown in Figure 8, where (a) and (b) are the echo signal waveforms at 200 in the azimuth direction and the fractional-order spectrum, respectively.

Set the sampling frequency to $18.468 \mathrm{MHz}$ and the number of sampling points to 400 . The fractional spectrum of the reconstructed signal with different sampling methods for the echo signal at 200 in the azimuthal direction is shown in Figure 9. As can be seen in Figure 8(a), the measured data contains a large amount of noise as well as quantization errors, so the relative errors used in the simulation experiments are hardly a valid measure of the reconstruction effect. For this purpose, the feature weights (FW) are introduced in this paper as follows:

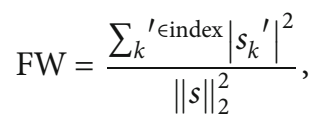

where $s$ is the coefficient vector of the signal under the fractional-order Fourier transform and $s_{k}{ }^{\prime}$ is the eigenfrequency coefficient in $s$. According to Figure 8(b), the coefficients in the fractional spectrum range of $[-13.53 \mathrm{MHz}$, $-12.88 \mathrm{MHz}]$ and $[-7.76 \mathrm{MHz},-7.53 \mathrm{MHz}]$ were selected as the eigenfrequency coefficients. The fractional spectrum of the reconstructed signals with different algorithms and the FW values are shown in Figure 9. The larger the FW value, the greater the proportion of useful echo components in the reconstructed signal and the better the imaging effect. From Figure 9, we can see that the FW value of the fractional spectrum of the reconstructed signal obtained by IFISTA is larger and the reconstruction effect is better.

After the reconstruction of each azimuthal signal is completed, imaging is performed using the distance Doppler algorithm, and to further quantify the imaging effect, image entropy [21] and image contrast [22] are introduced:

$$
\mathrm{IE}=\ln \left(\sum_{b \in \mathbb{R}} \sum_{a \in \mathbb{R}}\left|I_{b a}\right|^{2}\right)-\frac{\left[\sum_{b \in \mathbb{R}} \sum_{a \in \mathbb{R}}\left(\left|I_{b a}\right|^{2} \ln \left|I_{b a}\right|^{2}\right)\right]}{\left(\sum_{b \in \mathbb{R}} \sum_{a \in \mathbb{R}}\left|I_{b a}\right|^{2}\right)},
$$

$$
\mathrm{IC}=\frac{\sqrt{\operatorname{mean}\left(\left(I_{b a}-\operatorname{mean}\left(I_{b a}\right)\right)^{2}\right)}}{\operatorname{mean}\left(I_{b a}\right)},
$$

where $\mathbf{I}=\left(I_{b a}\right)_{b \in \mathbb{R}, a \in \mathbb{R}}$ represents the image and mean $(\cdot)$ is the mean operator. The lower the image entropy and the higher the image contrast, the better the imaging quality. The reconstruction effect under different reconstruction algorithms is shown in Figure 10.

As can be seen from the imaging results in Figure 10, the best imaging results are obtained after the reconstruction of the signal by the compression method proposed in this paper. The algorithm proposed in this paper is more conducive to retaining the useful information in the echo signal, so the IE value is smaller, the IC value is higher, and the reconstructed effect is better.

\section{Conclusions}

The application of broadband radar is becoming more and more widespread. We use compressed sensing to compress the echo data in order to solve the pressure on storage and transmission caused by the oversized data volume. In this paper, the IFISTA scheme is proposed to reconstruct the compressed sampled LFM echo signal. For the conventional FISTA algorithm, all the coefficients are approximated with the shrinkage operator, which will lose some useful information. To solve this problem, IFISTA is proposed to obtain a better reconstruction. The method is to first analyze the LFM signal during the iterative process and then construct the index vector to select the feature coefficients that are protected from shrinkage during the iterative process. Experimental analysis of simulated and real LFM echo signals shows that the IFISTA algorithm retains useful coefficients in the reconstruction of the LFM echo signal, reduces the loss of useful information, and allows for highly accurate echo signal reconstruction with fewer observations in the presence of sampling noise. The effectiveness and superiority of the algorithm proposed in this paper are verified through simulation and analysis of application examples.

\section{Data Availability}

The data used to support the findings of this study are available from the corresponding author upon request.

\section{Conflicts of Interest}

The authors declare that they have no conflicts of interest.

\section{Acknowledgments}

This work was funded by the National Natural Science Foundation of China (Grant No. 61501493). 


\section{References}

[1] H. Arab, S. Dufour, E. Moldovan, C. Akyel, and S. O. Tatu, "Accurate and robust CW-LFM radar sensor: transceiver front-end design and implementation," IEEE Sensors Journal, vol. 19, no. 5, pp. 1943-1950, 2019.

[2] H. Fan, L. Ren, E. Mao, Q. Liu, and J. Yang, "A high-precision phase-derived velocity measurement method for high-speed targets based on wideband direct sampling LFM radar," IEEE Transactions on Geoscience and Remote Sensing, vol. 57, no. 12, pp. 10147-10163, 2019.

[3] P. K. Kroh, R. Simon, and S. J. Rupitsch, "Classification of sonar targets in air-a neural network approach," Proceedings, vol. 2, no. 13, p. 929, 2018.

[4] D. L. Donoho, "Compressed sensing," IEEE Transactions on Information Theory, vol. 52, no. 4, pp. 1289-1306, 2006.

[5] R. G. Baraniuk, E. Candès, R. Nowak, and M. Vetterli, "Compressive sampling," IEEE Signal Processing Magazine, vol. 25, no. 2, pp. 1433-1452, 2008.

[6] Y. Qin, J. Zou, B. Tang, Y. Wang, and H. Chen, "Transient feature extraction by the improved orthogonal matching pursuit and K-SVD algorithm with adaptive transient dictionary," IEEE Transactions on Industrial Informatics, vol. 16, no. 1, pp. 215-227, 2020.

[7] E. J. Candes and T. Tao, "Decoding by linear programming," IEEE Transactions on Information Theory, vol. 51, no. 12, pp. 4203-4215, 2005.

[8] E. J. Candès and Y. Plan, "Near-ideal model selection by 1 minimization," Annals of Statistics, vol. 37, no. 5A, pp. 21452177, 2009.

[9] E. J. Candès, J. K. Romberg, and T. Tao, "Stable signal recovery from incomplete and inaccurate measurements," Communications on Pure and Applied Mathematics, vol. 59, no. 8, pp. 1207-1223, 2006.

[10] H. Li, Y. Ma, and Y. Fu, “An improved RIP-based performance guarantee for sparse signal recovery via simultaneous orthogonal matching pursuit," Signal Processing, vol. 144, pp. 29-35, 2018.

[11] D. Needell, C. A. Bouman, E. L. Miller, R. Vershynin, and I. Pollak, "Greedy signal recovery and uncertainty principles," Proceedings of SPIE-The International Society for Optical Engineering, vol. 6814, 2008.

[12] J. A. Tropp and A. C. Gilbert, "Signal recovery from random measurements via orthogonal matching pursuit," IEEE Transactions on Information Theory, vol. 53, no. 12, pp. 4655-4666, 2007.

[13] D. Needell and R. Vershynin, "Uniform uncertainty principle and signal recovery via regularized orthogonal matching pursuit," Foundations of Computational Mathematics, vol. 9, no. 3, pp. 317-334, 2009.

[14] M. S. Rao, K. K. Naik, and K. M. Reddy, "Radar signal recovery using compressive sampling matching pursuit algorithm," Defence Science Journal, vol. 67, no. 1, p. 94, 2016.

[15] A. Beck and M. Teboulle, "A fast iterative shrinkagethresholding algorithm for linear inverse problems," SIAM Journal on Imaging Sciences, vol. 2, no. 1, pp. 183-202, 2009.

[16] Y. Wang, J. Xiang, Q. Mo, and S. He, "Compressed sparse time-frequency feature representation via compressive sensing and its applications in fault diagnosis," Measurement, vol. 68, pp. 70-81, 2015.
[17] Y. Qin, "A new family of model-based impulsive wavelets and their sparse representation for rolling bearing fault diagnosis," IEEE Transactions on Industrial Electronics, vol. 65, no. 3, pp. 2716-2726, 2018.

[18] V. Namias, "The fractional order Fourier transform and its application to quantum mechanics," Geoderma, vol. 25, no. 3, pp. 241-265, 1980.

[19] H. M. Ozaktas, O. Arikan, M. A. Kutay, and G. Bozdagt, "Digital computation of the fractional Fourier transform," IEEE Transactions on Signal Processing, vol. 44, no. 9, pp. 21412150, 1996.

[20] I. G. Cumming and F. H. Wong, Digital Signal Processing of Synthetic Aperture Radar Data: Algorithms and Implementation, Artech House, 2004.

[21] L. Yang, M. Xing, L. Zhang, J. Sheng, and Z. Bao, "Entropybased motion error correction for high-resolution spotlight SAR imagery," IET Radar, Sonar \& Navigation, vol. 6, no. 7, pp. 627-637, 2012.

[22] M. Martorella, F. Berizzi, and B. Haywood, "Contrast maximisation based technique for 2-D ISAR autofocusing," IEE Proceedings-Radar Sonar and Navigation, vol. 152, no. 4, pp. 253-262, 2005. 